Research Article

\title{
Computation of Rayleigh Damping Coefficients for the Seismic Analysis of a Hydro-Powerhouse
}

\author{
Zhiqiang Song and Chenhui Su \\ State Key Laboratory Base of Eco-Hydraulic Engineering in Arid Area, Xian University of Technology, Xian 710048, China \\ Correspondence should be addressed to Zhiqiang Song; szhiq2004@126.com
}

Received 12 April 2017; Accepted 27 July 2017; Published 30 August 2017

Academic Editor: Xing Ma

Copyright (C) 2017 Zhiqiang Song and Chenhui Su. This is an open access article distributed under the Creative Commons Attribution License, which permits unrestricted use, distribution, and reproduction in any medium, provided the original work is properly cited.

\begin{abstract}
The mass and stiffness of the upper and lower structures of a powerhouse are different. As such, the first two vibration modes mostly indicate the dynamic characteristics of the upper structure, and the precise seismic response of a powerhouse is difficult to obtain on the basis of Rayleigh damping coefficients acquired using the fundamental frequencies of this structure. The damping ratio of each mode is relatively accurate when the least square method is used, but the accuracy of the damping ratios that contribute substantially to seismic responses is hardly ensured. The error of dynamic responses may even be amplified. In this study, modes that greatly influence these responses are found on the basis of mode participation mass, and Rayleigh damping coefficients are obtained. Seismic response distortion attributed to large differences in Rayleigh damping coefficients because of improper modal selection is avoided by using the proposed method, which is also simpler and more accurate than the least square method. Numerical experiments show that the damping matrix determined by using the Rayleigh damping coefficients identified by our method is closer to the actual value and the seismic response of the powerhouse is more reasonable than that revealed through the least square method.
\end{abstract}

\section{Introduction}

With the exploitation of water resources in southwest China, large-scale hydropower stations have been established in regions frequently hit by earthquakes. The antiseismic safety of powerhouses is essential for water turbine generators and operators in powerhouses. The seismic responses of powerhouse structures are often analyzed with time history method. For example, structural dynamic time history analysis includes modal superposition and step-by-step integration methods. Multi-degree-of-freedom vibration is decoupled in multiple single-degree-of-freedom vibrations in modal superposition method. Single-degree-of-freedom systems can be subjected to a precise dynamic response analysis through Duhamel's integration. With this method, input damping is accurate and high-speed calculation is achieved. However, modal superposition time history analysis method is only applicable to linear elastic structure systems because a structure possesses different decomposition modes at various times due to nonlinear structural material, nonlinear contact state, and other parameters. Therefore, a step-by-step integration dynamic time history analysis method can be used for a nonlinear system, which requires damping matrix establishment.

Unlike the formation of stiffness and mass matrixes, the formation mechanism of damping is complicated; that is, a damping matrix can be calculated by using a construction method but cannot be directly determined by identifying the material, size, and characteristics of structures [1]. Consequently, different damping matrix construction theories have been proposed [2-9]. For instance, a Rayleigh damping model is widely used because of its excellent advantages [1013]. (1) In this model, the damping matrix of a structure is a linear combination of mass and stiffness matrixes. As such, Rayleigh damping models can provide a clear physical meaning and present a convenient expression. Thus, these models can be easily applied. (2) A Rayleigh damping matrix must be orthogonal to mode shapes. Consequently, decoupling the dynamic equations of multiple degree-offreedom systems via mode superposition become convenient. 
Mode damping ratios can be directly used in single degreeof-freedom systems (generated by decoupling) dynamic response calculation. Therefore, damping input shows an enhanced accuracy and a reduced calculation scale. (3) Rayleigh damping coefficients can be determined by the orthogonality of a damping matrix for a modal shape. (4) With appropriate Rayleigh damping coefficients, results of a dynamic response analysis of a multi-degree-of-freedom system are the same as experimental data. A damping model is also embedded in commercial finite element software, and Rayleigh damping models are considered as a basis for damping matrix construction commonly utilized in the seismic time history analysis of hydraulic structures.

The damping matrix of a structure is the linear combination of the mass and stiffness matrixes of a Rayleigh damping model:

$$
[C]=\alpha[M]+\beta[K]
$$

where $\alpha$ and $\beta$, respectively, represent the mass and stiffness proportional damping coefficients, which are collectively known as Rayleigh damping coefficients. $[M],[C]$, and $[K]$ are the mass, damping, and stiffness matrixes, respectively. In traditional methods, two reference vibration modes $(i$ and $j$-order) are selected, and their damping ratios $\zeta_{i}$ and $\zeta_{j}$ obtained through measurement or reliable test data estimation and their frequencies $\omega_{i}$ and $\omega_{j}$ are used to calculate $\alpha$ and $\beta$ :

$$
\left\{\begin{array}{l}
\alpha \\
\beta
\end{array}\right\}=\frac{2 \omega_{i} \omega_{j}}{\omega_{j}^{2}-\omega_{i}^{2}}\left(\begin{array}{cc}
\omega_{j} & -\omega_{i} \\
-\frac{1}{\omega_{j}} & \frac{1}{\omega_{i}}
\end{array}\right)\left\{\begin{array}{l}
\zeta_{i} \\
\zeta_{j}
\end{array}\right\}
$$

This equation can be simplified as follows when $\zeta_{i}=\zeta_{j}=\zeta$ :

$$
\left\{\begin{array}{l}
\alpha \\
\beta
\end{array}\right\}=\frac{2 \zeta}{\omega_{i}+\omega_{j}}\left\{\begin{array}{c}
\omega_{i} \omega_{j} \\
1
\end{array}\right\}
$$

Two orders of the reference frequency can be easily and appropriately selected to determine Rayleigh damping coefficients when the degree of freedom of a structure is low or the dynamic response of this structure is controlled by some low-order modes. For example, the first two orders are generally obtained as reference frequencies in traditional methods. For complex structures and structures with a number of modes that contribute greatly to dynamic responses, difficulties in selecting two orders of reference frequencies to obtain reasonable Rayleigh damping coefficients $\alpha$ and $\beta$ are encountered. If damping coefficients are chosen inappropriately, a slight difference in damping may seriously distort the calculation of the seismic response of a given structure [1417].

Many scholars investigated the calculation of Rayleigh damping coefficients. Pan et al. [18] proposed a constrained optimization method to determine Rayleigh damping coefficients for the accurate analysis of complex structures. An objective function is defined as a complete quadratic combination of the modal errors of a peak base reaction evaluated through response spectral analysis. An optimization constraint is enforced to determine the exact damping ratio of modes that contribute greatly to dynamic responses. This method is based on Duhamel's integral formula, which is suitable for linear elastic systems. Yang et al. [19] studied the application of a multi-mode-based computation method in single-layer cylindrical latticed shells because the traditional two-mode Rayleigh damping method is unsuitable. Yang et al. [19] also suggested that the multi-mode-based computation method is preferable when many dominant modes are distributed loosely and found in a wide range of frequencies under some ground motions. Jehel et al. [20] comprehensively compared the initial structural stiffness and updated tangent stiffness of Rayleigh damping models to allow a practitioner to objectively choose the type of Rayleigh damping models that satisfy his needs and be provided with useful analytical tools for the design of these models with good control on their damping ratios during inelastic analysis. Erduran [21] evaluated the effects of a Rayleigh damping model based on the engineering demand parameters of two steel moment-resisting frame buildings. Rayleigh damping models, which combine mass and stiffness proportional components, are anchored at reduced modal frequencies, which create reasonable damping forces and floor acceleration demands for both buildings but do not suppress higher-mode effects. Zhe et al. [22] developed an improved method to calculate Rayleigh damping coefficients for the seismic response time history analysis of powerhouse structures by considering the spectrum characteristics of the ground motion and the frequency characteristics of these structures. Using the improved method, Zhe et al. [22] obtained calculation results that are consistent with experimental findings.

Hongshi [23] initially compared and analyzed several methods of calculating Rayleigh damping coefficients and subsequently proposed the least square method to minimize the difference between the calculated damping ratio and the actual damping ratio within the cutoff frequency. Li et al. [24] then established the corresponding method for the seismic response analysis of powerhouse structures by calculating the proportional damping coefficient through the weighted least square method.

Analyzing the seismic dynamic response of long-span arch bridges and super high structures with long periods, Lou $[15,25]$ found that Rayleigh damping coefficients determined by traditional methods involving the first two orders of frequencies as reference frequencies inaccurately reflect the actual damping effect of long-period structures in a dynamic process and even create a large deviation. Hence, calculation methods of the Rayleigh damping coefficients of long-period structures in dynamic processes should be further discussed.

The definition of long- and short-period structures is a relative concept depending on the relationship between the basic period of a structure and the characteristic period of dynamic loads. A short-period structure is characterized as a structure whose basic period is less than or close to the 
characteristic period of external loads. Otherwise, a given structure is called a long-period structure. The basic characteristic period is usually $10^{-1} \mathrm{~s}$ or higher because the stiffness of the upper frame structure is relatively weak. Therefore, this weak structure is also described as a long-period structure because of the characteristic period of seismic waves. The mass and stiffness of the upper and lower structures of powerhouses are different. As such, the first two modes of vibration often indicate the dynamic characteristics of the upper structure. The damping matrix obtained by traditional methods of calculating Rayleigh damping coefficients does not easily reveal the actual damping of the whole powerhouse structure. Hence, the influence of the calculation methods of Rayleigh damping coefficients on the dynamic response of a powerhouse under seismic actions should be investigated. In this study, the calculation of Rayleigh damping coefficients is examined to analyze the seismic responses of a powerhouse.

To calculate Rayleigh damping coefficients, Chopra [1] suggested that "in dealing with practical problems, it is reasonable to select the modes of vibrations $i$ and $j$ with specific damping ratios to ensure that the damping ratios of all modes of vibration that contribute greatly to the dynamic response are reasonable." Differences in the mass and stiffness of the upper and lower structures of a powerhouse remarkably create the dynamic characteristics of a powerhouse structure. The first two vibration modes often involve the relatively soft upper structure of the powerhouse, whose mode participation mass is quite smaller than that of the whole powerhouse. The damping of these buildings under seismic actions is mainly due to various interior frictions and deformations of components and the ones between them. Therefore, mode participation mass should be considered as a key factor affecting the calculation of damping. The modes that contribute greatly to dynamic responses are found on the basis of mode participation mass. In this study, the Rayleigh damping coefficient is calculated.

This paper is organized as follows. Section 2 introduces the finite element models of a powerhouse and several methods of calculating Rayleigh damping coefficients. The two modes that remarkably affect the dynamic responses are determined on the basis of mode participation mass, and the Rayleigh damping coefficients are calculated. Section 3 presents the results of the dynamic response obtained by different methods. Numerical results show that the proposed method accurately reveals the two modes contributing to the dynamic response of the powerhouse, and the calculated Rayleigh damping coefficients are consistent with actual results. The calculated results are also closer to the exact solutions and even higher than those acquired by the least square method. Section 4 provides the conclusion.

\section{Seismic Analysis Model of Powerhouses and Calculation Method of Rayleigh Damping Coefficients}

2.1. Three-Dimensional Finite Element Model and Seismic Inputs. In Figure 1, the three-dimensional finite element model of a typical unit of the hydropower station is established. The depth of the foundation is about twice the

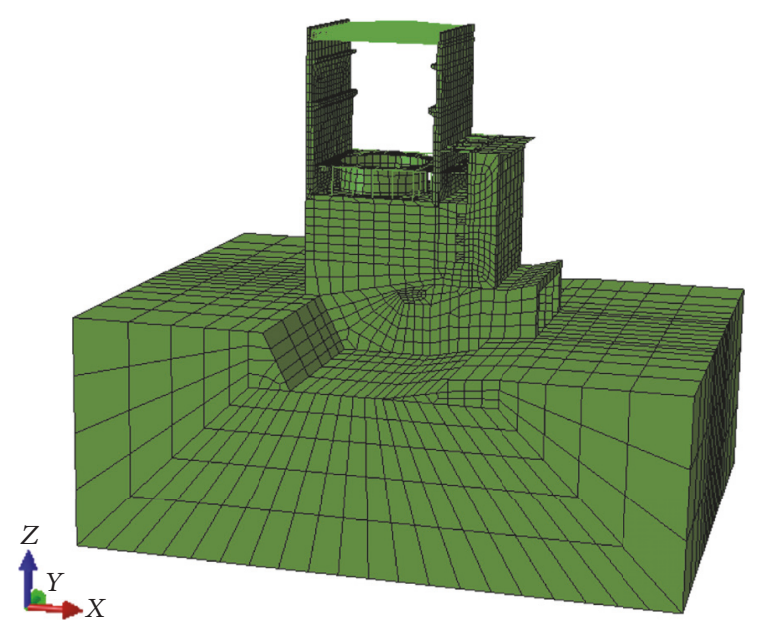

FIGURE 1: Finite element model of the powerhouse.

height of the powerhouse, and the upstream and downstream and the left and right sides extend twice the height of the powerhouse because of the elastic coupling effect of the foundation. The foundation boundary condition is that the bottom is fixed and the four boundaries are normally constrained. Turbine generators, cranes, roof loads, and hydrodynamic pressures are simulated as the additional mass at corresponding locations. A linear elastic model is used in the structure, and the local strengthening effects of the volute steel plate and the seating ring are neglected. The damage cracks of the concrete around the volute are also disregarded. Zhang et al. [26] observed that cracks on thin parts of the concrete of a volute greatly change the local stiffness of the volute but slightly alter the displacement, speed, and acceleration of the upper structure compared with those without cracks. The nonmass foundation, which does not affect the response peaks of the structure, is adopted for calculation convenience.

In engineering designs, a single seismic time history does not generally influence the dynamic response of a structure. Different factors, such as site characteristics, fortification intensity, and probability of ground motion, should be considered in accordance with the requirements of the antiseismic codes of various types of structures. Therefore, seismic time history is simulated in this paper according to the response spectrum of the standard design in literature [27].

Under actual conditions, the site type is $I_{0}$, the characteristic period Ts is $0.02 \mathrm{~s}$, the representative of the maximum value of the response spectrum of the standard design is 2.25 , the seismic intensity is increased from degrees 7 to 8 according to requirements of the grade and importance of structures, the peak acceleration of the horizontal ground motion is increased from $0.05 \mathrm{~g}$ to $0.1 \mathrm{~g}$, the time step of the ground motion is $0.01 \mathrm{~s}$, and the total time is $20 \mathrm{~s}$. Figure 2 shows the generated seismic time histories, in which the correlation coefficients of $x$ and $y$ directions, $x$ and $z$ directions, and $y$ and $z$ directions are 0.0011, 0.1737, and 0.0002 , respectively. These coefficients are less than 0.3 , which 


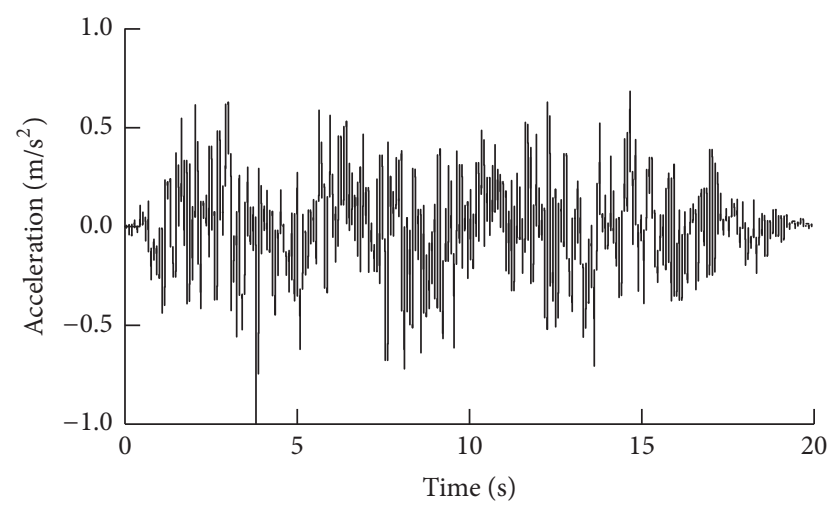

(a) $x$-direction

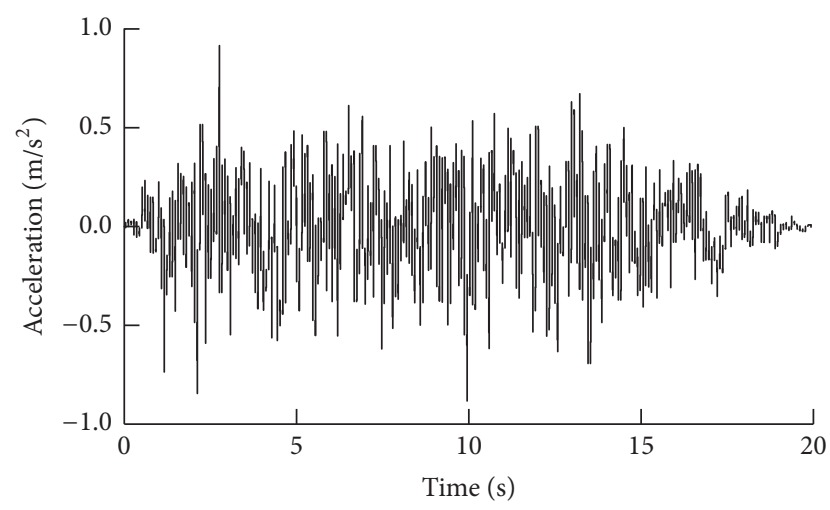

(b) $y$-direction

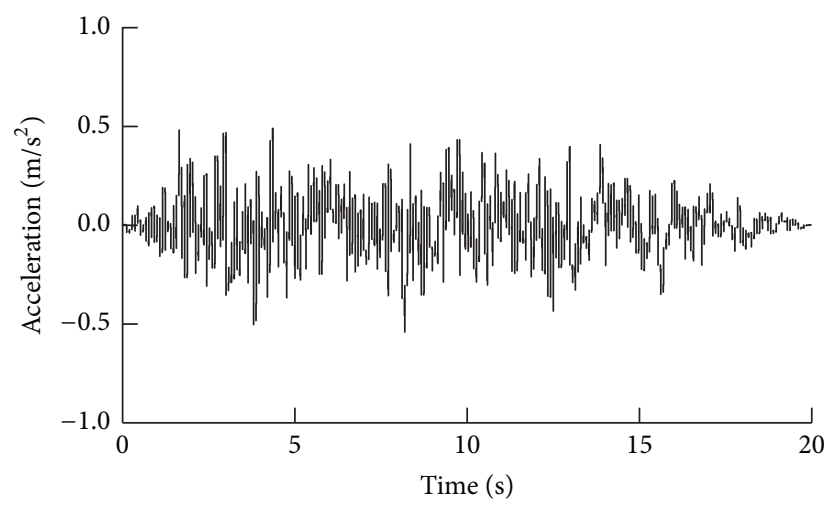

(c) $z$-direction

FIGURE 2: Calculation of the time history of seismic acceleration.

satisfies the requirements [26] that the time histories in the three directions should be independent of one another.

\subsection{Calculation Method of Rayleigh Damping Coefficients.} A reasonable proportional damping matrix based on the Rayleigh damping model is necessary when the step-by-step integration method is used to conduct dynamic time history analysis. In this case, Rayleigh damping coefficients directly affect the results of dynamic analysis.

A linear elastic powerhouse structure is presented as an example and the response of the modal superposition time history method (referred to as modal method thereafter) is used as a reference, which is the standard of measurement, to evaluate the influence of various Rayleigh damping coefficients obtained by different methods on the seismic response in the step-by-step integration method. The relevant specification [27] requires that the damping ratio of the powerhouse under the action of ground motion is 0.07 , which is substituted into the following Rayleigh damping coefficients to obtain the corresponding damping matrix of each method and then applied to the step-by-step integration method to solve the seismic response of the powerhouse.

The finite element model of the powerhouse is initially analyzed to determine the natural frequency of some orders of the structure, which is required for the calculation of Rayleigh damping coefficients. The first 80 orders are obtained. The mode participation masses of $x, y$, and $z$ directions are $92.1 \%, 90.8 \%$, and $91.0 \%$ of the total mass of the model, respectively. These values are more than $90 \%$ of the total mass of the model and thus satisfy the requirement of calculation accuracy. Figure 3 illustrates some orders of the vibration modes of the hydro-powerhouse and the typical vibration characteristics of the ground-type powerhouse structure. The frequencies are low and dense, and the frequencies of the first 10 orders range from $0.55 \mathrm{~Hz}$ to $6.21 \mathrm{~Hz}$. The natural period of the vibration of the first order is $1.83 \mathrm{~s}$, which is much larger than the characteristic period of a seismic wave, and this finding indicates that the powerhouse is a long-period structure. The first-order vibration mode is mainly the vibration of the frame columns and roof grids in the upstream and downstream directions. Most of the modal shapes of the low orders are mainly the bending and torsional vibration of the upper frame structure and the vibration of the weak parts of the generator floor and windshield. The twoorder vibration modes with the largest mode participation masses of the three directions are the 13th $(7.72 \mathrm{~Hz})$ and $25 \mathrm{th}(12.81 \mathrm{~Hz})$, whose mode participation masses are $14.6 \%$ and $25.3 \%$, respectively. The vibration modes correspond to the whole vibration of the powerhouse and the higher-order bending vibration of the superstructure.

According to the natural frequency of the structure of the powerhouse and the characteristic of each mode of vibration, 


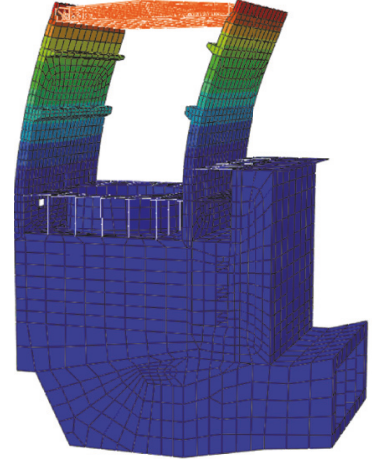

(a) First order

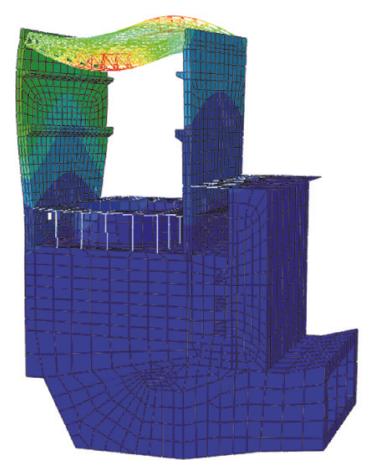

(e) Fifth order

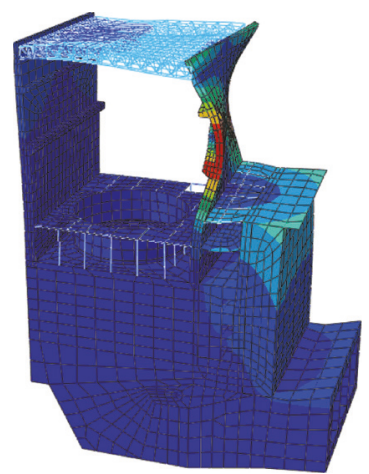

(i) Ninth order

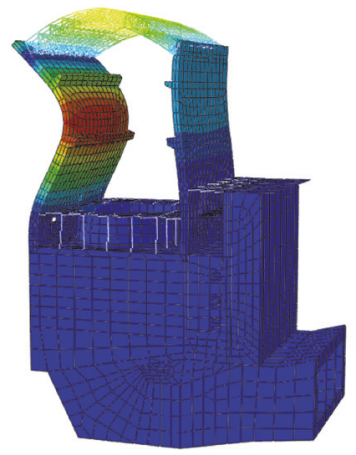

(b) Second order

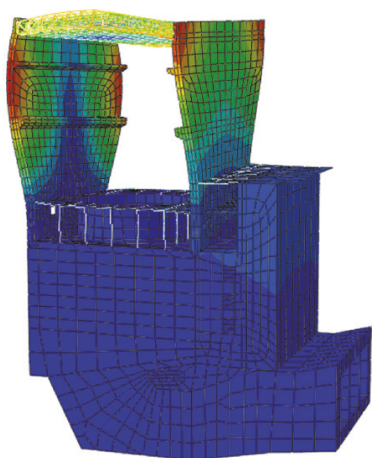

(f) Sixth order

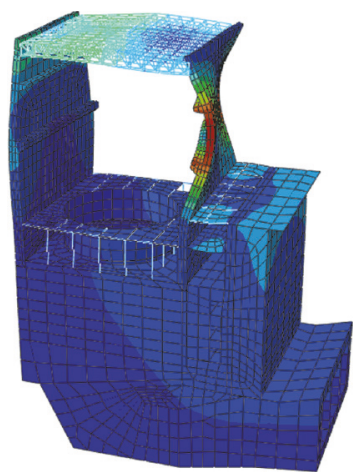

(j) Tenth order

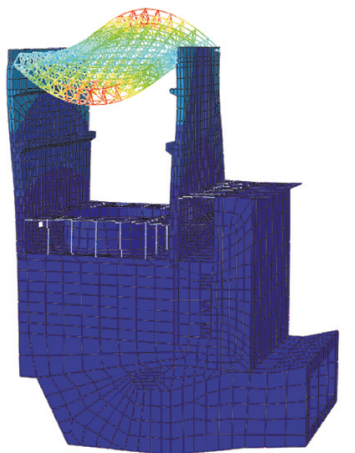

(c) Third order

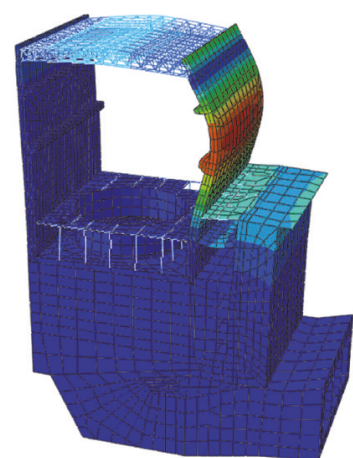

(g) Seventh order

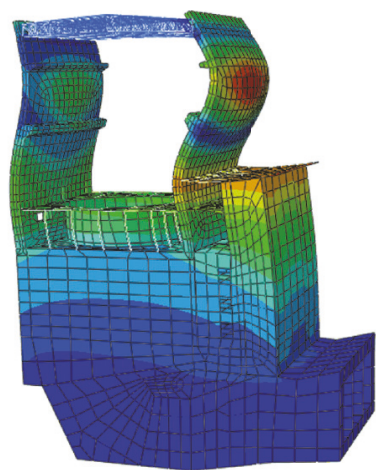

(k) Thirteenth order

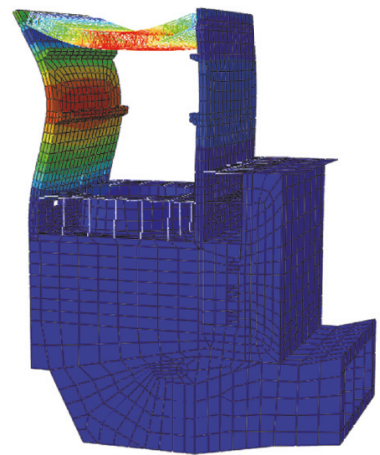

(d) Fourth order

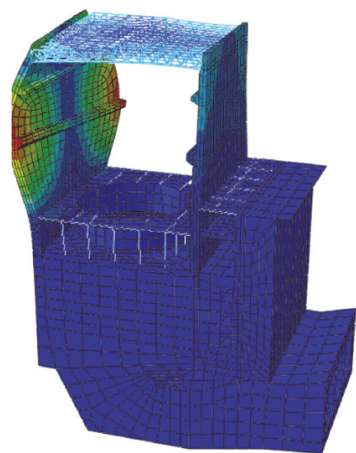

(h) Eighth order

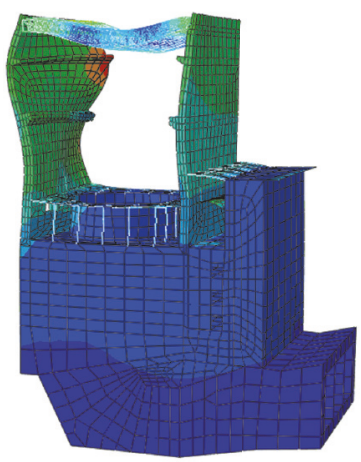

(l) Twenty-fifth order

Figure 3: Vibration modes of the hydro-powerhouse.

the methods widely used and the proposed calculation method of Rayleigh damping coefficients can be summarized as follows:

(1) In method 1 or the traditional method, the first two orders of natural frequency are used to determine Rayleigh damping coefficients. This method is based on the assumption that low-order vibration modes contribute greatly to structural dynamic responses. Many orders of vibration modes are also complex, and low-order modes mainly correspond to the vibrations of the weak parts of the upper structure. Therefore, this assumption is inapplicable. The damping ratios of the vibration modes obtained in this method are too large, but the first two orders are equal to the actual values. Consequently, the contributions of the thirdorder vibration modes to dynamic responses become weakened and induce a low total structural response. Designing an antiseismic powerhouse based on these response results is risky. The first two natural frequencies are 0.55 and $2.96 \mathrm{~Hz}$ according to the calculation of the finite element structure of the powerhouse. The damping coefficients obtained by method 1 are $\alpha_{1}=0.4053$ and $\beta_{1}=0.0064$ (3).

(2) In method 2, the frequency band of interest is selected; that is, the natural frequencies of the first order and the last order (80th order) are used as the parameters. The mode participation masses of $x, y$, and $z$ directions are larger than $90 \%$ of the total mass of the model and thus satisfy the calculation accuracy requirements. The natural frequency of the 80 th order is $27.96 \mathrm{~Hz}$. According to (3), the damping coefficients acquired by this method are $\alpha_{2}=0.4709$ and $\beta_{2}$ $=0.0008$.

According to the calculation principle of Rayleigh damping coefficients, the frequencies $\omega_{i}$ and $\omega_{j}$ of the two constants 
TABLE 1: Rayleigh damping coefficients obtained by different methods.

\begin{tabular}{lcccc}
\hline Methods & $\alpha$ & $\beta$ & According to & Involved orders \\
\hline Modal methods & $\backslash$ & $\backslash$ & Constant damping ratio: 0.07 & $1-80$ \\
Case 1 & 0.4053 & 0.0064 & First and second orders & 1,2 \\
Case 2 & 0.4709 & 0.0008 & First and last orders & 1,80 \\
Case 3 & 0.9553 & 0.0010 & Optimization by least square method & $1-80$ \\
Case 4 & 0.5286 & 0.0012 & Weighted least squares, weight coefficients: $1 / \omega_{i}$ & $1-80$ \\
Case 5 & 0.4064 & 0.0063 & Weighted least squares, weight coefficients: $\exp \left(-\omega_{i}\right)$ & $1-80$ \\
Case 6 & 4.2364 & 0.0011 & Two orders have largest mode participation masses & 13,25 \\
Case 7 & 0.7878 & 0.0015 & Weighted least squares, weight coefficients: $m_{i} / M$ & $1-80$ \\
\hline
\end{tabular}

$\alpha$ and $\beta$ should cover the target frequency band in the structural analysis. The calculation of the specific frequency band should be considered in terms of the frequency components of the loads acting on a particular structure and the dynamic characteristics of this structure.

However, this method may cause several problems. (1) The selection of target frequency band relies on experience. (2) The set damping ratios within the band may be too small. As such, the calculated structural response may be excessively large and inappropriate if the deviation is overly large.

(3) In method 3, the least square method without weighting coefficients is used. The damping coefficients are calculated by applying the least square method to determine the least square sum of the difference between the calculated damping ratio of each order and the actual damping ratio within the cutoff frequency. The formula is expressed as follows:

$$
\min _{\alpha, \beta} \sum_{i=1}^{n}\left(\frac{\alpha}{2 \omega_{i}}+\frac{\beta \omega_{i}}{2}-\zeta\right)^{2} .
$$

(4) In method 4, given weighting coefficients, the orders that contribute greatly to the damping effect will become more dominant, while the ones that contribute a little are less important. The objective function is

$$
\min _{\alpha, \beta} \sum_{i=1}^{n} \frac{1}{\omega_{i}}\left(\frac{\alpha}{2 \omega_{i}}+\frac{\beta \omega_{i}}{2}-\zeta\right)^{2} .
$$

The weighting coefficient is the reciprocal of the natural frequency of each order. Therefore, low orders correspond to high weight coefficients and contribute to the dynamic responses of structures.

(5) Method 5 is almost the same as method 4. These methods differ in terms of their weight coefficients; that is, the weight coefficient of method 5 is the negative exponential function of the natural frequency of each order:

$$
\min _{\alpha, \beta} \sum_{i=1}^{n} \exp \left(-\omega_{i}\right)\left(\frac{\alpha}{2 \omega_{i}}+\frac{\beta \omega_{i}}{2}-\zeta\right)^{2} .
$$

(6) Method 6 is our proposed approach. The orders that contribute greatly to the responses are selected on the basis of mode participation mass. The two orders with the largest total mode participation masses in the three directions are considered as references to calculate the damping coefficients.
According to the results of the self-vibration characteristics of the structure of the powerhouse, these two orders are the 13th $(7.72 \mathrm{~Hz})$ and $25 \mathrm{th}(12.81 \mathrm{~Hz})$, and their masses are $14.6 \%$ and $25.3 \%$, respectively.

(7) Method 7 is based on method 6, and the least square method involving mode participation masses as coefficients is established and expressed as follows:

$$
\min _{\alpha, \beta} \sum_{i=1}^{n} \frac{m_{i}}{M}\left(\frac{\alpha}{2 \omega_{i}}+\frac{\beta \omega_{i}}{2}-\zeta\right)^{2} .
$$

The mode orders and damping coefficients obtained by each method are shown in Table 1.

\section{Seismic Analysis of Different Rayleigh Damping Coefficients Obtained Using Various Calculation Methods for the Powerhouse}

Five typical points in the powerhouse are selected to reveal the representative seismic response of the powerhouse comprehensively: 8079 in the middle of the top of the downstream wall, 6636 in the middle of the joint between the generator floor and the downstream wall, 8255 in the mid-span of the generator floor, 8088 in the downstream side of the cover fan, and 2889 in the downstream side of the seating ring. The relative peak acceleration of each typical point determined by different calculation methods is listed in Table 2 , and the direction of acceleration is disregarded and the maximum absolute value is considered. The peak acceleration of each point acquired by the modal superposition method is set as the standard value. The results of methods 1 to 7 are normalized and the percentages of deviation from the results acquired by the modal superposition method are obtained (Figure 4).

In Table 2 and Figure 4, different calculation methods of damping coefficients significantly affect the calculation results of the seismic response of the powerhouse.

In method 1, the relative peak acceleration of each typical point in different directions is smaller than the exact values because the damping ratios of the 1st and 2 nd orders of the vibration modes are equal to 0.07 and the damping ratios of the 3 rd to the 80 th orders are greater than 0.07 . These findings indicate that damping ratios increase significantly as the order increases and thus yield high damping ratios and 
TABLE 2: Peak accelerations of typical points determined by different methods $\left(\mathrm{m} / \mathrm{s}^{2}\right)$.

\begin{tabular}{|c|c|c|c|c|c|c|c|c|c|}
\hline Node & Direction & Modal method & Case 1 & Case 2 & Case 3 & Case 4 & Case 5 & Case 6 & Case 7 \\
\hline \multirow{3}{*}{2889} & $x$ & 0.482 & 0.423 & 0.732 & 0.623 & 0.603 & 0.424 & 0.483 & 0.535 \\
\hline & $y$ & 1.368 & 1.239 & 1.835 & 1.680 & 1.675 & 1.245 & 1.437 & 1.617 \\
\hline & $z$ & 0.442 & 0.380 & 0.834 & 0.725 & 0.707 & 0.383 & 0.558 & 0.641 \\
\hline \multirow{3}{*}{6636} & $x$ & 2.947 & 2.193 & 5.407 & 4.665 & 4.642 & 2.208 & 3.133 & 4.108 \\
\hline & $y$ & 2.186 & 1.996 & 2.898 & 2.522 & 2.595 & 1.999 & 2.112 & 2.357 \\
\hline & $z$ & 1.360 & 0.852 & 2.333 & 2.031 & 1.929 & 0.860 & 1.559 & 1.706 \\
\hline \multirow{3}{*}{8079} & $x$ & 3.971 & 2.807 & 7.310 & 5.983 & 5.944 & 2.829 & 4.179 & 5.139 \\
\hline & $y$ & 4.163 & 3.457 & 4.971 & 4.902 & 4.913 & 3.475 & 3.892 & 4.831 \\
\hline & $z$ & 2.844 & 1.534 & 4.898 & 4.459 & 4.354 & 1.555 & 3.395 & 3.901 \\
\hline \multirow{3}{*}{8088} & $x$ & 1.919 & 1.541 & 3.349 & 2.999 & 2.989 & 1.551 & 2.212 & 2.707 \\
\hline & $y$ & 1.864 & 1.744 & 2.757 & 2.421 & 2.465 & 1.747 & 1.811 & 2.239 \\
\hline & $z$ & 1.167 & 0.792 & 1.703 & 1.556 & 1.534 & 0.800 & 1.233 & 1.422 \\
\hline \multirow{3}{*}{8255} & $x$ & 2.830 & 2.141 & 5.207 & 4.501 & 4.483 & 2.156 & 3.030 & 3.971 \\
\hline & $y$ & 2.168 & 1.967 & 2.955 & 2.557 & 2.622 & 1.971 & 2.077 & 2.370 \\
\hline & $z$ & 1.666 & 1.060 & 2.536 & 2.301 & 2.243 & 1.069 & 1.798 & 2.060 \\
\hline
\end{tabular}

Note. $x$ represents the upstream and downstream direction, $y$ shows the direction perpendicular to the stream direction, and $z$ denotes the vertical direction.

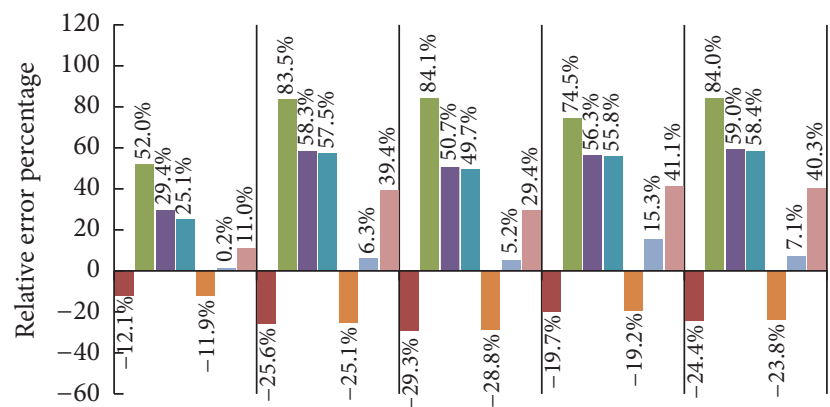

Node 2889 Node 6636 Node 8079 Node 8088 Node 8255 Node number

Case 1

Case 2

Case 3

Case 4

$$
\begin{array}{r}
\text { Case } 5 \\
\text { Case } 6 \\
\text { Case } 7
\end{array}
$$

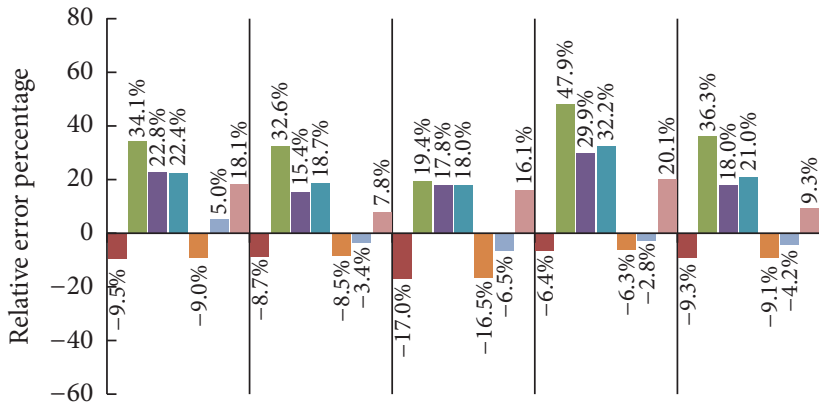

Node 2889 Node 6636 Node 8079 Node 8088 Node 8255

Node number

$\begin{array}{ll}\text { Case } 1 & \text { Case } 5 \\ \text { Case } 2 & \text { Case } 6\end{array}$

$\begin{array}{ll}\text { Case } 2 & \text { Case } 6 \\ \text { Case } 3 & \text { Case } 7\end{array}$

Case 4

(b) $y$-direction

(a) $x$-direction

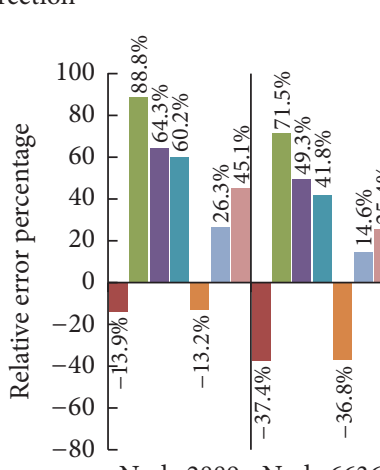

Node 2889 Node 6636 Node 8079 Node 8088 Node 8255

Node number

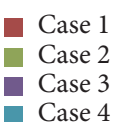

$$
\begin{array}{r}
\text { Case } 5 \\
\text { Case } 6 \\
\text { Case } 7
\end{array}
$$

(c) $z$-direction

FIGURE 4: Error of the peak acceleration. 


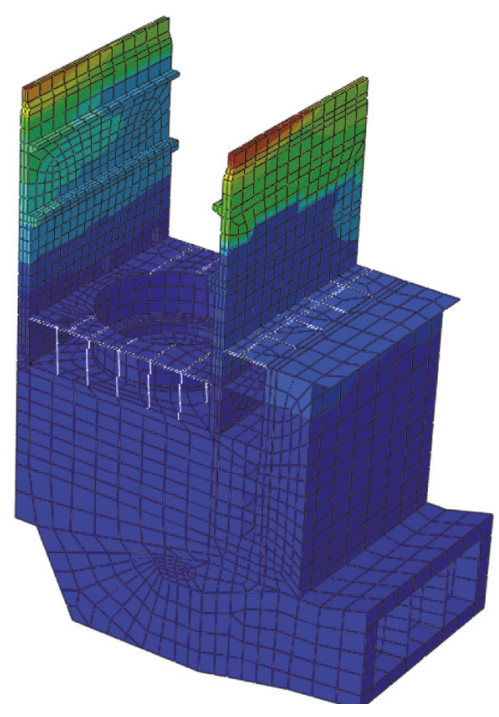

(a) $x$-direction

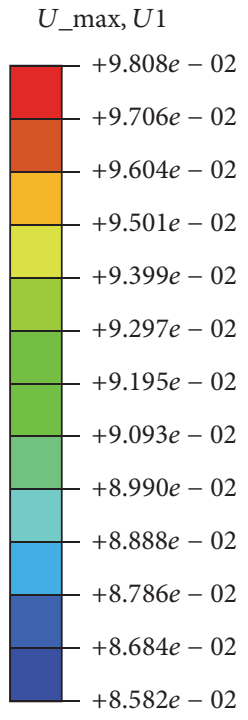

FIGURE 5: Displacement envelope figures of the whole powerhouse.

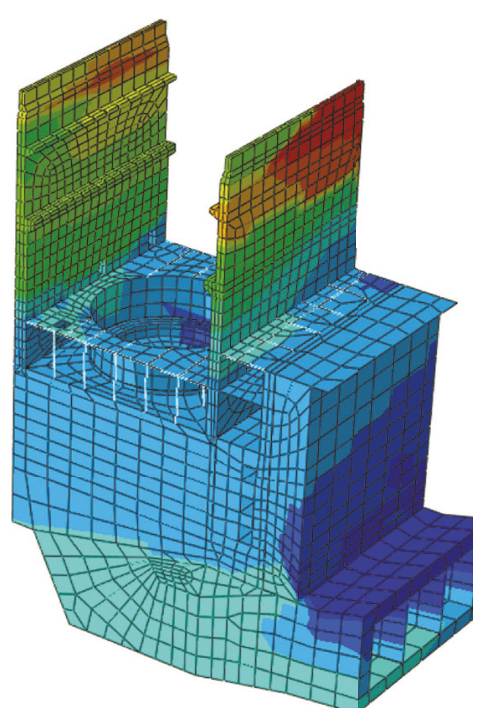

(b) $y$-direction

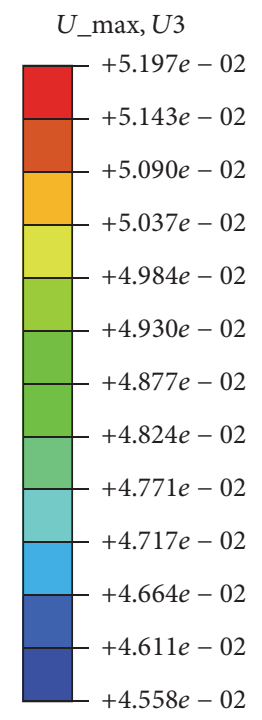

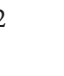

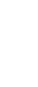


In method 7, the damping coefficients are obtained on the basis of the weighted least square method. In contrast to methods 4 and 5, method 7 considers the mode participation masses as the weighting coefficients; that is, the orders that contribute greatly to the dynamic response are selected on the basis of the mode participation mass. The damping coefficients of most of the modes obtained by this method are closer to the exact values, whereas the damping coefficients of the modes that contribute greatly to the dynamic response are less accurate. The relative peak acceleration of each typical point in different directions is closer to the exact solution determined by method 7 than those obtained by methods 2-5 but less accurate than the one acquired by method 6 .

The relative peak acceleration of each typical point in different directions obtained by methods $3,4,5$, and 7 , which were based on the least square method, is closer to the exact value than that identified by methods 1 and 2 . Therefore, the solutions provided by the least square method or the weighted least square method are more accurate than those established by methods 1 and 2. Methods 4 and 5 are based on the weighted least square method and on the assumption that low-order modes contribute greatly to seismic responses. However, the results calculated by the two methods differ greatly. In particular, the response of the powerhouse acquired by method 4 is greater than the exact value, whereas the response determined by method 5 is smaller than the exact finding. The relative peak acceleration of point 8079 detected by method 4 is also 2.8 times greater than that revealed by method 5 . The mode participation mode is thus regarded as an important factor influencing the damping coefficients of the powerhouse in methods 6 and 7 . By comparison, the solutions from method 7 are less accurate than those from method 6.

These comparisons and analyses reveal that the drawbacks of the least square method and the weighted least square method are inevitable. On the one hand, the weight coefficients remarkably influence the calculation results. Therefore, the damping coefficients of all orders can be considered to reduce the uncertainty caused by the two chosen modes, but the uncertainty attributed to the selection of the weight coefficients cannot be removed. On the other hand, we have yet to verify importance of the orders that contribute greatly to the response during calculation to obtain the optimal objective function in the least square method and the weighted least square method. The accuracy of the damping ratios of the orders that remarkably contribute may be severely reduced. Therefore, the least squares method and the weighted least squares method are slightly uncontrollable and should be used cautiously in practical engineering.

Table 3 and Figure 6 show the relative peak displacements for each typical point obtained by different methods, in which the direction of displacement is disregarded and the maximum absolute value is considered, and the percentage of deviation from values obtained by modal superposition method. The relative peak displacements of the points in different directions are similar to those of the relative peak acceleration analyzed in the preceding sections. Only few values obtained by methods 1 and 5 are closer to the exact values because changes in damping affect the distribution of the relative displacements of the structure in time history under the same seismic input. As such, peak displacements are influenced cumulatively. Overall, the results determined by method 6 are still the closest solutions to the exact values because they are more stable and reliable than those identified by other methods. In the example, the largest deviations from the exact relative peak acceleration determined by methods $1-7$ in different directions are $-28.39 \%, 63.61 \%, 41.59 \%$, $41.44 \%, 27.83 \%, 30.37 \%$, and $28.67 \%$, respectively.

The solutions obtained by method 6 are slightly larger than and closer to the exact values. The modes that contribute greatly to the dynamic response are found on the basis of the mode participation mass, and the damping coefficients are calculated using method 6 . These findings are consistent with the calculation method of Rayleigh damping coefficients proposed by Chopra [1]: "In dealing with practical problems, it is reasonable to select the modes of vibration $i$ and $j$ with specific damping ratios to ensure that damping ratios of all modes of vibration that contribute greatly to the dynamic response are reasonable." Therefore, we verify that mode participation mass is related to the contribution of orders to seismic responses. Our numerical experiments demonstrate that the Rayleigh damping coefficients determined by this method are closer to the actual values and the seismic response of the structure is more reasonable.

\section{Conclusions}

Calculation methods of Rayleigh damping coefficients greatly affect the results of the seismic responses of powerhouses and buildings with similar structural characteristics. In method 1 or the traditional method, the damping ratios except the first two values are larger than the exact ratios. As a result, the structural dynamic response is significantly smaller. The first two vibration modes often indicate the dynamic characteristics of the upper structure, not the whole structure. The damping ratios of most of the vibration modes in the middle are smaller than the exact values. As such, the structural dynamic response in method 2 is significantly larger than that in method 1. To obtain the optimal objective function, we can use the least square method and the weighted least square method for an accurate calculation of damping coefficients. However, whether the orders that contribute greatly to responses play an important role in the calculation remains uncertain. The errors of the damping ratios of the orders with a significant contribution may be severely increased. Thus, the dynamic response of a structure is unable to satisfy the required accuracy in practical engineering.

In the method proposed in this study, the mode orders that influence the dynamic responses are found on the basis of mode participation mass, and Rayleigh damping coefficients are determined by using the natural frequencies of the two mode orders, whose mode participation masses are the largest. The seismic response distortion attributed to large differences in Rayleigh damping coefficients caused by improper modal selection is avoided by using the proposed method, which is also simpler and more accurate than the least square method. Our numerical experiments show that the damping matrix determined by using Rayleigh damping coefficients in this method is closer to the actual value, and the seismic response of the powerhouse is more acceptable. 
TABLE 3: Peak displacements of each point obtained by different methods $(\mathrm{cm})$.

\begin{tabular}{|c|c|c|c|c|c|c|c|c|c|}
\hline Node & Direction & Modal method & Case 1 & Case 2 & Case 3 & Case 4 & Case 5 & Case 6 & Case 7 \\
\hline \multirow{3}{*}{2889} & $x$ & 0.020 & 0.022 & 0.027 & 0.026 & 0.025 & 0.022 & 0.026 & 0.024 \\
\hline & $y$ & 0.121 & 0.112 & 0.138 & 0.137 & 0.136 & 0.113 & 0.133 & 0.134 \\
\hline & $z$ & 0.016 & 0.015 & 0.025 & 0.022 & 0.022 & 0.015 & 0.019 & 0.020 \\
\hline \multirow{3}{*}{6636} & $x$ & 0.134 & 0.120 & 0.214 & 0.187 & 0.187 & 0.120 & 0.173 & 0.170 \\
\hline & $y$ & 0.191 & 0.188 & 0.230 & 0.209 & 0.213 & 0.188 & 0.218 & 0.206 \\
\hline & $z$ & 0.033 & 0.026 & 0.054 & 0.046 & 0.045 & 0.026 & 0.037 & 0.040 \\
\hline \multirow{3}{*}{8079} & $x$ & 6.882 & 7.287 & 7.429 & 6.164 & 7.116 & 7.289 & 7.739 & 6.431 \\
\hline & $y$ & 0.527 & 0.502 & 0.597 & 0.583 & 0.580 & 0.503 & 0.541 & 0.573 \\
\hline & $z$ & 0.302 & 0.307 & 0.326 & 0.278 & 0.308 & 0.308 & 0.317 & 0.287 \\
\hline \multirow{3}{*}{8088} & $x$ & 0.093 & 0.082 & 0.143 & 0.129 & 0.128 & 0.082 & 0.118 & 0.117 \\
\hline & $y$ & 0.164 & 0.157 & 0.194 & 0.180 & 0.181 & 0.157 & 0.184 & 0.177 \\
\hline & $z$ & 0.028 & 0.021 & 0.043 & 0.038 & 0.038 & 0.021 & 0.032 & 0.034 \\
\hline \multirow{3}{*}{8255} & $x$ & 0.129 & 0.116 & 0.208 & 0.182 & 0.182 & 0.116 & 0.168 & 0.166 \\
\hline & $y$ & 0.186 & 0.183 & 0.228 & 0.205 & 0.212 & 0.183 & 0.212 & 0.201 \\
\hline & $z$ & 0.042 & 0.030 & 0.065 & 0.058 & 0.058 & 0.030 & 0.045 & 0.052 \\
\hline
\end{tabular}

Note. $x$ represents the upstream and downstream direction, $y$ corresponds to the direction perpendicular to the stream direction, and $z$ indicates the vertical direction.

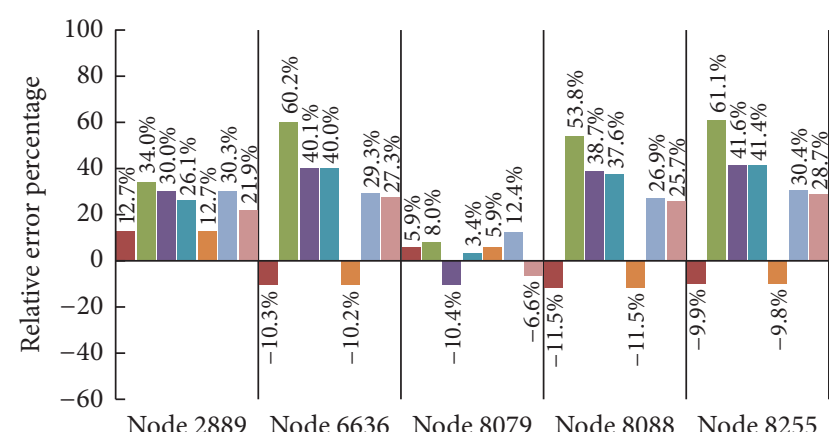

Node number

Case 1

Case 2

Case 3

Case 4

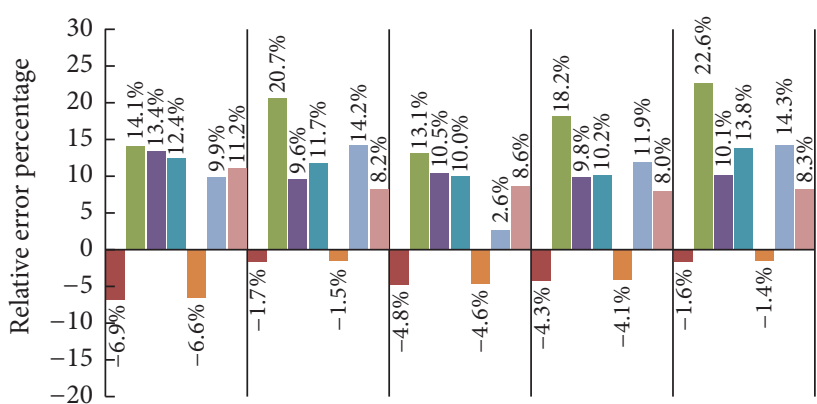

Node 2889 Node 6636 Node 8079 Node 8088 Node 8255 Node number

$\begin{array}{ll}\text { Case } 1 & \text { Case } 5 \\ \text { Case } 2 & \text { Case } 6 \\ \text { Case } 3 & \text { Case 7 }\end{array}$

(b) $y$-direction

Case 4

(a) $x$-direction

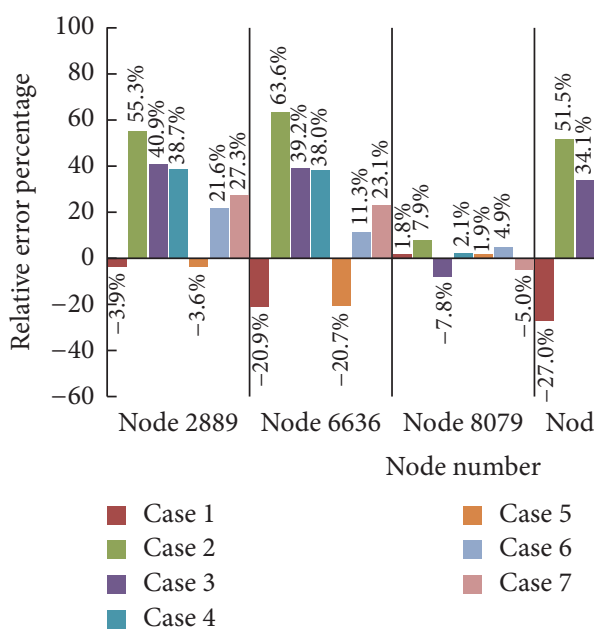

(c) $z$-direction

FIGURE 6: Error of peak displacements. 


\section{Conflicts of Interest}

The authors declare that there are no conflicts of interest regarding the publication of this paper.

\section{Acknowledgments}

This research was supported by the National Natural Science Foundation of China (51479165) and State Key Laboratory Base of Eco-Hydraulic Engineering in Arid Area Independent Research Project (2016ZZKT-1).

\section{References}

[1] A. K. Chopra, Dynamics of structures: Theory and applications to earthquake engineering, Upper Saddle River: Prentice Hall, 2011.

[2] T. K. Caughey and M. E. O’Kelly, "Classical normal modes in damped linear dynamic systems," vol. 32, pp. 583-588, 1965.

[3] Z. Liang and G. C. Lee, "Representation of damping matrix," Journal of Engineering Mechanics, vol. 117, no. 5, pp. 1005-1020, 1991.

[4] H. Zongming, B. Shaoliang, and L. Ming, "Review on the damping in earthquake response time-history analysis of structures," Earthquake Engineering and Engineering Vibration, vol. 16, no. 2, pp. 95-102, 1996.

[5] J. Dong, H. Z. Deng, and Z. M. Wang, "Studies on the damping models for structural dynamic time history analysis," World Information on Earthquake, vol. 16, pp. 63-69, 2000.

[6] S. Adhikari, "Damping modelling using generalized proportional damping," Journal of Sound and Vibration, vol. 293, no. 1-2, pp. 156-170, 2006.

[7] D. Danhui and S. Limin, "Damping modeling and evaluation of structural dynamic finite element analysis," Journal of Vibration and Shock, vol. 26, no. 2, pp. 121-124, 2007.

[8] J. E. Luco, "A note on classical damping matrices," Earthquake Engineering and Structural Dynamics, vol. 37, no. 4, pp. 615-626, 2008.

[9] H.-D. Zhang and Y.-F. Wang, "Study on seismic time-history response of structures with complex damping," Gongcheng Lixue/Engineering Mechanics, vol. 27, no. 1, pp. 109-115, 2010.

[10] D. R. Pant, A. C. Wijeyewickrema, and M. A. ElGawady, "Appropriate viscous damping for nonlinear time-history analysis of base-isolated reinforced concrete buildings," Earthquake Engineering \& Structural Dynamics, vol. 42, no. 15, pp. 23212339, 2013.

[11] J. Wang, "Rayleigh coefficients for series infrastructure systems with multiple damping properties," JVC/Journal of Vibration and Control, vol. 21, no. 6, pp. 1234-1248, 2015.

[12] R. E. Spears and S. R. Jensen, "Approach for selection of rayleigh damping parameters used for time history analysis," Journal of Pressure Vessel Technology, vol. 134, no. 6, 2012.

[13] Y. Li, Y. Sun, B. Li, and Z. Xu, "Penalty function-based method for obtaining a reliability indicator of gravity dam stability," Computers and Geotechnics, vol. 81, pp. 19-25, 2017.

[14] American Society of Civil Engineers, Seismic Analysis of SafetyRelated Nuclear Structures and Commentary, ASCE 4-98 Standard, 2000.

[15] L. Menglin, S. Lei, and S. Fei, "Effect s of damping matrix modeling on seismic response of super high-rise structures," Structural Engineers, vol. 29, no. 1, pp. 55-61, 2013.
[16] D.-G. Zou, B. Xu, and X.-J. Kong, "Study of influence of different methods for calculating Rayleigh damping coefficient on high earth-rock dam seismic response," Yantu Lixue/Rock and Soil Mechanics, vol. 32, no. 3, pp. 797-803, 2011.

[17] D.-G. Pan and L.-L. Gao, "Comparison of determination methods for Rayleigh damping coefficients and effects on seismic responses of structures," Gongcheng Lixue/Engineering Mechanics, vol. 32, no. 6, pp. 192-199, 2015.

[18] D. G. Pan, G. D. Chen, and L. L. Gao, "A constrained optimal Rayleigh damping coefficients for structures with closely spaced natural frequencies in seismic analysis," Advances in Structural Engineering, vol. 20, no. 1, pp. 81-95, 2017.

[19] D.-B. Yang, Y.-G. Zhang, and J.-Z. Wu, "Computation of Rayleigh damping coefficients in seismic time-history analysis of spatial structures," Journal of the International Association for Shell and Spatial Structures, vol. 51, no. 164, pp. 125-135, 2010.

[20] P. Jehel, P. Léger, and A. Ibrahimbegovic, "Initial versus tangent stiffness-based Rayleigh damping in inelastic time history seismic analyses," Earthquake Engineering and Structural Dynamics, vol. 43, no. 3, pp. 467-484, 2014.

[21] E. Erduran, "Evaluation of Rayleigh damping and its influence on engineering demand parameter estimates," Earthquake Engineering and Structural Dynamics, vol. 41, no. 14, pp. 1905-1919, 2012.

[22] L. Zhe, W. Gongxian, and H. Yong, "Application of improved calculation method of Rayleigh damping coefficients to seismic response analysis on quay crane structure," Journal of South China University of Technology Natural Science Edition, vol. 43, no. 6, pp. 103-109, 2015.

[23] L. Hongshi, "Relative errors and determination of Rayleigh damping scale coefficient," Journal of Hunan institute of Engineering, vol. 11, no. 3-4, pp. 36-38, 2001.

[24] X.-J. Li, C.-L. Hou, R. Pan, G.-L. Zhou, and Y. Yang, "Effect of damping matrix selection on seismic response of nuclear power plant structures," Zhendong yu Chongji/Journal of Vibration and Shock, vol. 34, no. 1, pp. 110-116, 2015.

[25] L. Menglin and Z. Jing, "Discussion on damping models for seismic response analysis of long-span bridge," Journal of Vibration and Shock, vol. 28, no. 5, pp. 22-25, 2009.

[26] Y.-L. Zhang, Z.-Y. Ma, Y. Wang, and J. Chen, "Effect of cracks on dynamics characteristics of hydropower house," Journal of Hydraulic Engineering, vol. 39, no. 8, pp. 982-986, 2008.

[27] National Energy Administration of China, Code for seismic design of hydraulic structures of hydropower project, NB 350472015, 2015. 


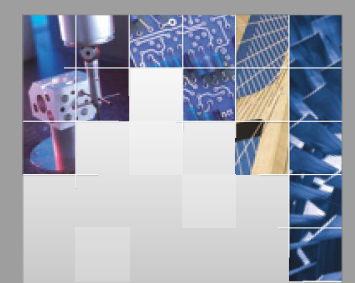

\section{Enfincering}
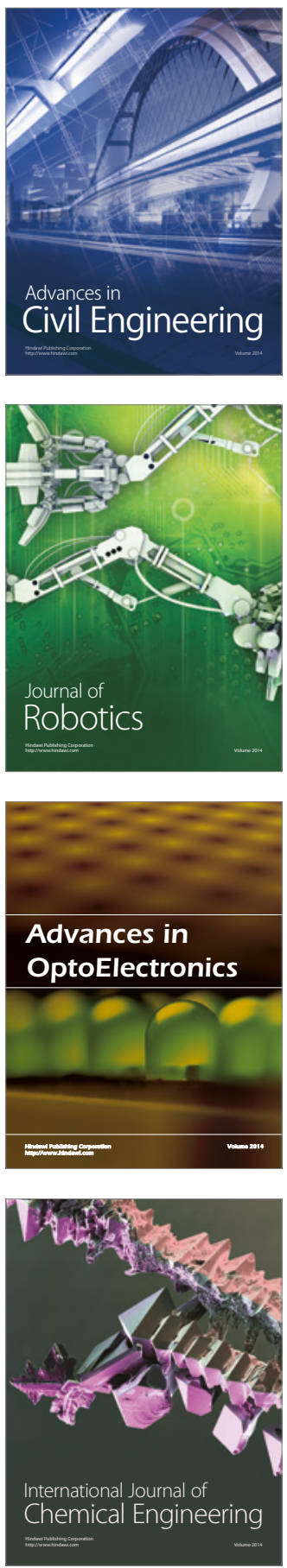

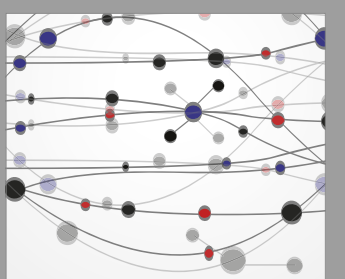

The Scientific World Journal

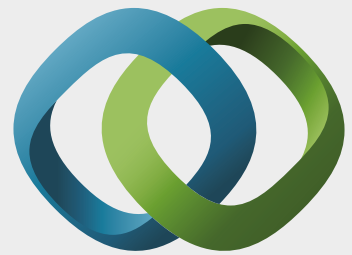

\section{Hindawi}

Submit your manuscripts at

https://www.hindawi.com
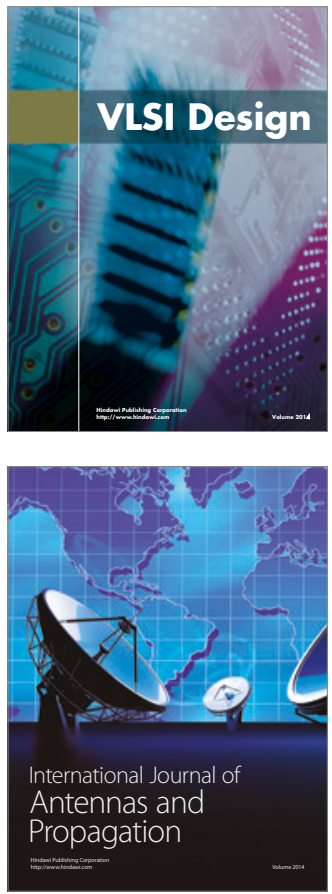

\section{Rotating}

Machinery
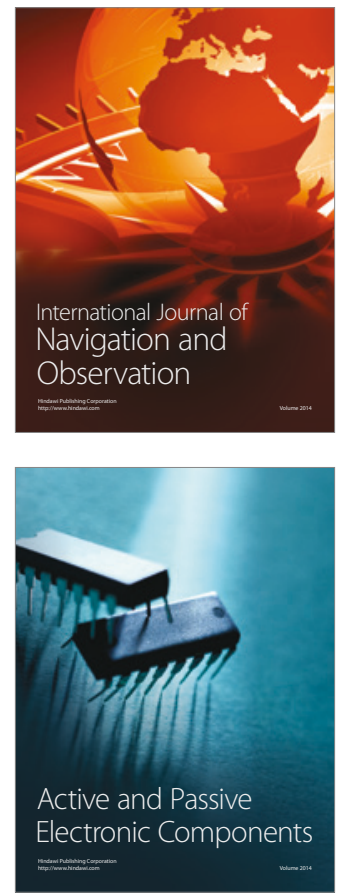
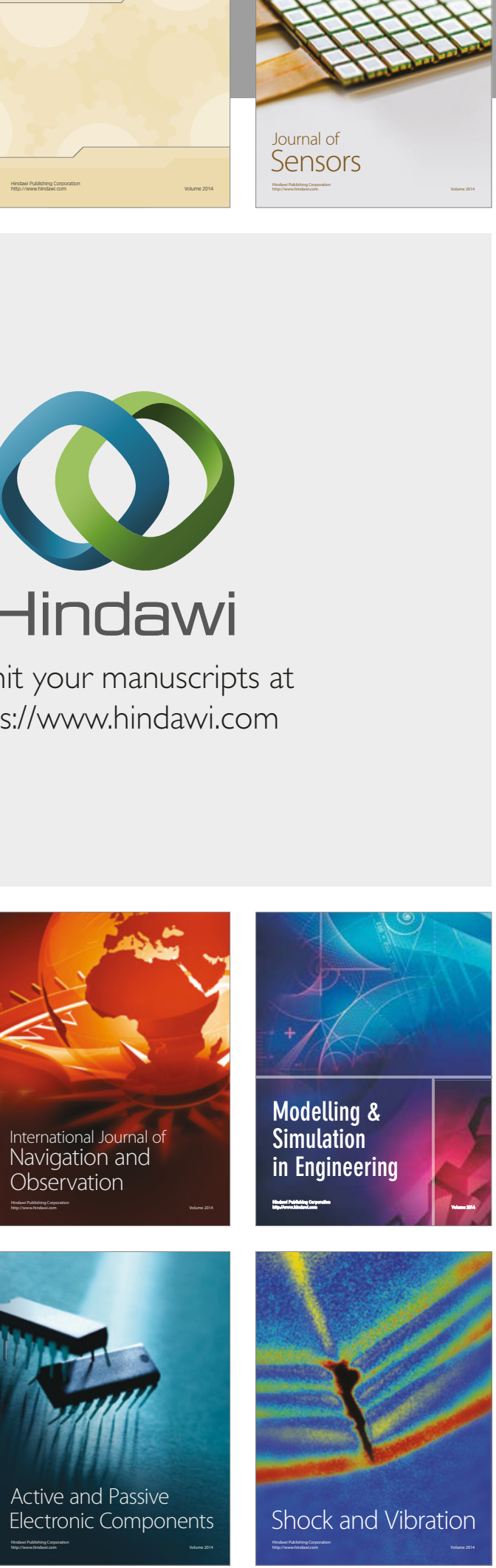
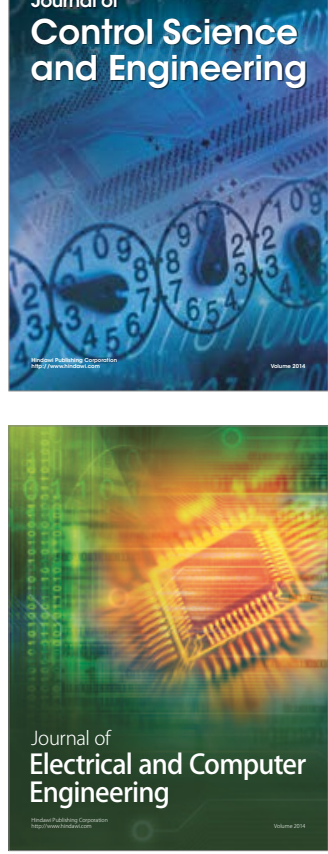

Distributed

Journal of

Control Science

and Engineering
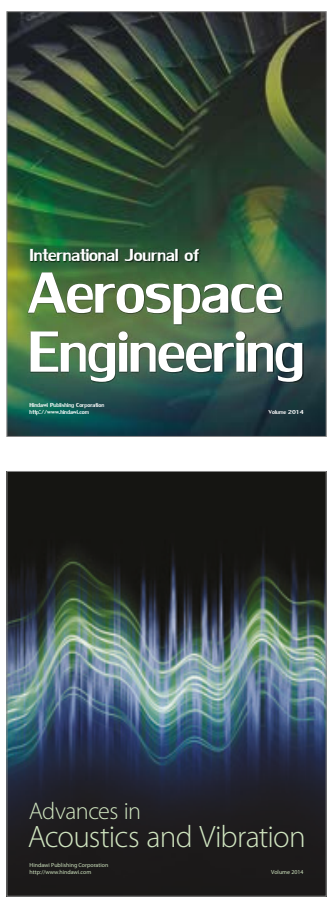

Sensor Networks 